\title{
Cost effectiveness of transoral robotic surgery for the treatment of oropharyngeal squamous cell carcinoma: a systematic review
}

This article was published in the following Dove Press journal:

Robotic Surgery: Research and Reviews

17 April 2015

Number of times this article has been viewed

\section{Nicole L Aaronson \\ Paul D Neubauer \\ Benjamin L Judson}

Yale Otolaryngology, Department of Surgery, Yale University School of Medicine, New Haven, CT, USA
Correspondence: Benjamin L Judson Yale Otolaryngology, Department of Surgery, Yale University School of Medicine, 333 Cedar Street,

PO Box 20804I, New Haven,

CT 06520, USA

Tel +l 2037852593

Fax +I 2037853970

Email benjamin.judson@yale.edu

\begin{abstract}
Since the first transoral use of the da Vinci robotic surgical system in 2005 and the subsequent FDA approval for the system's use in 2009, there has been a large uptick in the number of transoral robotic surgery (TORS) procedures performed in the United States. The most common use of TORS has been in the treatment of oropharyngeal squamous cell carcinoma (OPSCC). The rise in TORS cases for OPSCC is driven in part by the epidemic of human papilloma virus (HPV)-associated OPSCC. The advantage of TORS is that it allows transoral resection of tumors previously requiring open approaches associated with longer hospitalization and recovery, and higher functional morbidity. In addition to oncologic and functional outcomes, the cost of treatments using the robot is also a consideration in determining what role TORS should have in the treatment of OPSCC. A systematic review of the literature was performed by searching for articles addressing the cost-effectiveness of TORS. We have analyzed the articles obtained and report that analysis here. The results include case series and other analyses. They suggest that TORS for OPSCC is cost-effective compared to other available modalities due to decreased hospital stay, increased chance of finding an unknown primary and thereby avoiding some adjuvant treatment, and decreased rates of gastrostomy tube and tracheotomy. The methods used for calculating costs varied widely, and studies universally lack long-term follow-up. Further studies are needed, which define and measure costs and compare TORS with open surgery and non-surgical treatments.
\end{abstract}

Keywords: oropharyngeal cancer, transoral robotic surgery, da Vinci robot, cost

\section{Introduction}

The da Vinci robotic system was first used for transoral surgery (TOS) in 2005 and has since been rapidly adopted for the excision of tumors in the upper aerodigestive tract (UADT), particularly in the oropharynx. ${ }^{1,2}$ In 2009, the FDA approved transoral robotic surgery (TORS) for benign lesions and T1-T2 lesions of the UADT. Compared to open surgical approaches for oropharyngeal squamous cell carcinoma (OPSCC), TORS allows per-oral surgical resection of the primary tumor, avoiding slow recovery and high morbidity associated with traditional surgery. In some situations, TORS also allows decreased use of chemoradiotherapy or radiotherapy, which are frequently employed as a definitive for OPSCC.

To satisfy patient demands for the newest technology, hospitals may face pressure to expand the use of their robotic system to include excision of tumors of the UADT, allowing them to maximize the use of a da Vinci system already used for urologic and gynecologic procedures. ${ }^{3}$ As a result of striving for better outcomes and expanded uses 
for emerging technologies, the use of TORS in head and neck cancer is growing and will likely continue to grow. In addition, the incidence of oropharyngeal cancer has increased due to human papilloma virus (HPV)-associated oropharyngeal cancer, further driving the need for improvements in treatment modalities. ${ }^{4}$

When evaluating a new treatment, parameters that should be considered include oncologic and functional outcomes, quality of life, and cost. The TORS series published report at least equivalent oncologic outcomes and better functional and quality-of-life outcomes compared to conventional surgical and non-surgical treatment approaches, ${ }^{5,6}$ although these results remain somewhat controversial and randomized studies evaluating these outcomes are underway. Given the concern over rising health care costs, which equaled $17.9 \%$ of the gross domestic product in the United States in 2012, the cost-effectiveness of new treatments must be carefully considered. ${ }^{7}$ Since the introduction of TORS, few studies have looked at its cost. This paper evaluates the current literature on the cost-effectiveness of TORS for oropharyngeal cancer.

\section{Methods}

A systematic search was conducted in PubMed for research papers on the cost effectiveness of TORS in OPSCC (Table 1). Relevant synonyms for the search terms "cost," "transoral robotic surgery," and “oropharyngeal cancer" were included. Original study data were included as well as systematic reviews. Opinion papers, animal studies, case reports, and studies not in English were excluded. Studies regarding urologic and gynecologic procedures were also excluded. Additionally, references of included articles were reviewed in order to identify any references not identified in the initial literature search.

\section{Results}

In total, ten relevant articles were retrieved. One article was excluded because it was not in English. Five others were

Table I PubMed MEDLINE search terms

\begin{tabular}{lll}
\hline aerodigestive & transoral surgery & cost utility \\
oropharyngeal carcinoma & robotics & cost \\
oropharyngeal cancer & robotic & cost analysis \\
$\begin{array}{l}\text { oropharyngeal neoplasm } \\
\text { neoplasm, unknown primary } \\
\text { base of tongue }\end{array}$ & TORS & \\
\hline
\end{tabular}

Notes: $(((($ aerodigestive[tiab]) OR (((oropharyngeal carcinoma*[tiab]) OR oropharyngeal cancer*[tiab]) OR ((“"Oropharyngeal Neoplasms”[Mesh]) OR "Neoplasms, Unknown Primary"[Mesh]) OR "base of tongue")))) AND ((transoral surgery[tiab]) OR (((“"Robotics”[Mesh]) OR robotic*[tiab]) OR tors[tiab]) OR "transoral robotic surgery"))) AND ((cost utility[tiab]) OR ((cost*[tiab]) OR (“Costs and Cost Analysis"[Mesh]))).

Abbreviation: TORS, transoral robotic surgery. excluded as they were not cost analyses of TORS. After these exclusions, four articles remained (Table 2). Cross-reference checking resulted in no additional references.

\section{Data analysis}

Moore et al performed a case series on cost analysis comparing TOS, including transoral laser microsurgery and TORS with concomitant neck dissection (ND), TOS with adjuvant radiation therapy (TOS + RT), TOS with adjuvant chemoradiation therapy (TOS + CRT), and primary chemoradiation therapy (CRT). Cost was defined as the money actually paid by the third-party payer or patient in 90 days after diagnosis of oropharyngeal cancer. It was found that while patients treated with surgery alone tended to have smaller tumors, their treatment was the least expensive $(\$ 37,435$ for private payers $/ \$ 15,664$ for government payers). Primary CRT was the most expensive modality $(\$ 198,285$ for private payers $/ \$ 57,429$ for government payers). CRT was even more expensive than TOS + CRT. $^{8}$

Richmon et al and Hammoudi et al examined the cost of TORS compared to open surgery in retrospective analyses. ${ }^{2,9}$ Richmon analyzed 9,601 patients, 116 of whom had TORS. Cost was calculated by multiplying total inpatient charges by all-payer inpatient cost-to-charge ratio from the Center for Medicare and Medicaid Services (CMS). ${ }^{2}$ Hammoudi et al included 26 patients in each group in France. This study defined cost as the average operating room (OR) cost multiplied by the OR time (cost of surgery) added to the average daily cost of an otolaryngology patient's hospitalization multiplied by the number of days in the hospital (cost of hospital stay). ${ }^{9}$

Both Richmon and Hammoudi's studies concluded that TORS was associated with fewer tracheotomies, less tube feeding, and decreased cost. Richmon determined that TORS was associated with a significantly decreased length of stay (LOS) (1.5 days less than open surgery) and decreased cost (\$4,285 less than open surgery). None of the TORS patients underwent gastrostomy tube placement or tracheotomy, whereas $19 \%$ of open surgery patients had gastrostomy tubes, and 36\% had tracheotomies. ${ }^{2}$ In Hammoudi's analysis TORS had a higher OR cost $(\$ 7,781 \vee \$ 4,375, P<0.001)$ but the total cost was significantly lower $(\$ 20,885$ v $\$ 27,926$, $P=0.03$ ) when compared to conventional surgery. The cost savings was due to shorter LOS in the TORS group versus conventional surgery ( 11 days v 19 days, $P=0.001){ }^{9}$

Byrd et al conducted a retrospective chart review of the cost effectiveness of TORS for the unknown primary. This paper did not address oropharyngeal tumors specifically but is relevant in the setting of increasing HPV-related cancers 


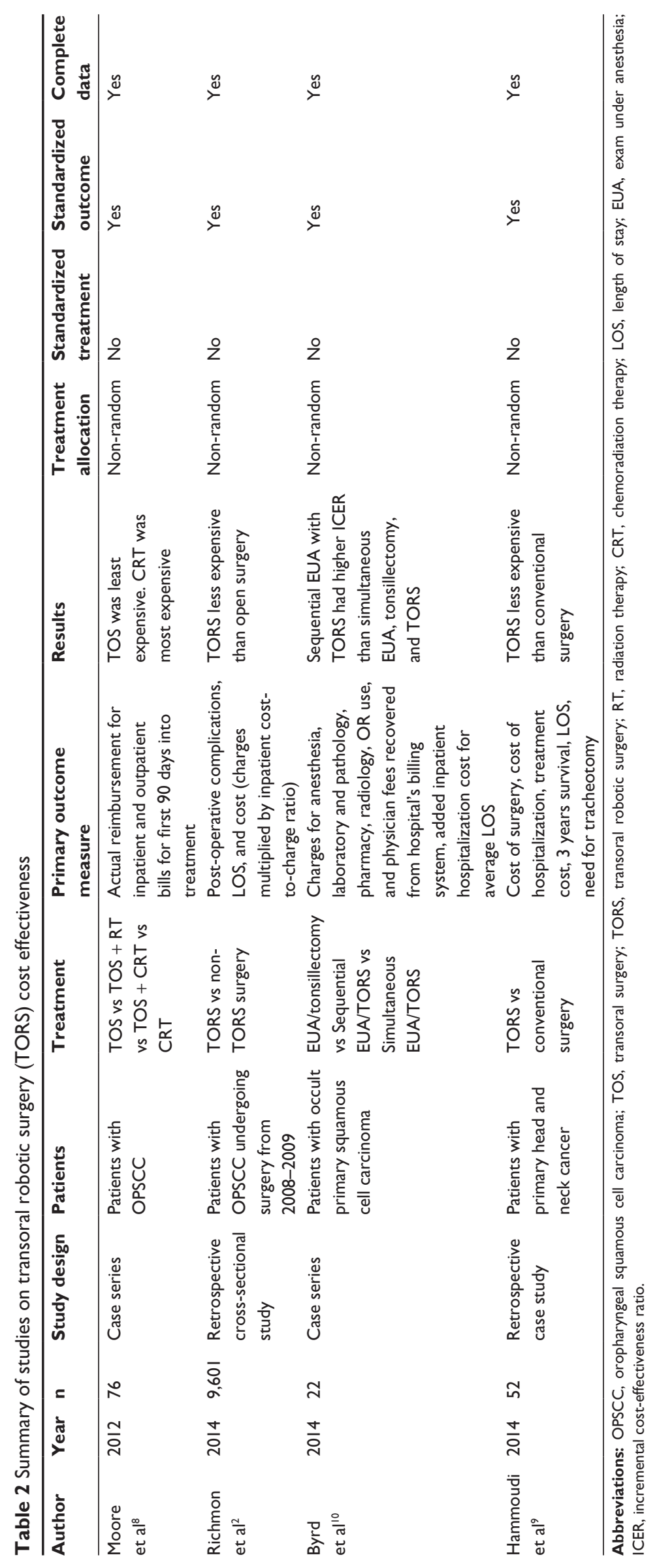


presenting as occult oropharyngeal primary tumors. Patients with unknown primaries were divided into three treatment groups: 1) exam under anesthesia (EUA) with tonsillectomy, 2) staged TORS base-of-tongue (BOT) resection after previous EUA/tonsillectomy and 3) simultaneous EUA, tonsillectomy, and TORS BOT resection. Nine of eleven primary tumors were identified in the sequential tonsillectomy/TORS group. Using the previously reported $30 \%$ identification rate of unknown primary tumors with EUA/tonsillectomy, the authors calculated that $87 \%$ of unknown primary tumors would be identified by adding a staged TORS BOT resection. For the simultaneous tonsillectomy/TORS group, 100\% of primary tumors were identified (6/6). ${ }^{10}$

Costs-to-charge ratios were used to calculate anesthesia, pathology, radiology, and laboratory charges. Average OR cost and average cost of hospitalization were calculated. The calculation of costs did not include fixed hospital costs, including the purchasing and amortization of the robot. The incremental cost-effectiveness ratio (ICER), which represents the cost to find each additional unknown primary, was calculated to be $\$ 8,619$ for sequential tonsillectomy and TORS, and \$5,774 for simultaneous EUA, tonsillectomy, and TORS. By identifying the primary tumor site, post-operative radiation may be avoided or at least limited in its field, further reducing the overall cost of treatment. ${ }^{10}$

\section{Discussion}

\section{Methods of calculation}

There is wide variation in how cost is defined in various studies, making comparisons between them difficult. Moore's case series provides the most accurate measure of cost by calculating the hospital's actual revenue for each treatment group, including percutaneous gastrostomy tube, specialty consultations, and subsequent admissions for medical issues over a three-month period, rather than estimating the cost. ${ }^{8}$ Billing practices vary widely between hospitals and insurance plans; hence, charges created by the hospital may not accurately reflect actual expenditures. Nevertheless, this case series does not consider patients' missed wages due to recovery time. IfTORS reduces a patient's time in the hospital and hence out of work, it may further decrease the cost to the patient by minimizing his or her lost wages.

\section{Length of stay}

LOS for TORS is consistently shorter compared to that for open surgery, and this is a major factor contributing to its increased cost effectiveness. Moore found a shorter number of inpatient days with TOS (2.4 days for TOS v 6.8 days for
CRT); however, TORS was not evaluated separately from other transoral approaches. ${ }^{8}$ Richmon found that the average LOS was 1.5 days less than that in the case of conventional surgery. ${ }^{2}$ In France, the average TORS LOS for oropharyngeal cancer was 11 days versus 19 days for conventional surgery. ${ }^{9}$ A retrospective analysis of 91 patients showed the mean LOS following TORS for all indications to be 1.5 days. ${ }^{14}$

\section{Capital equipment}

The cost of capital equipment bears inclusion when calculating the cost effectiveness of TORS. The da Vinci robot was estimated to cost $\$ 1.5$ million in 2010, with the need for an annual service contract for greater than $\$ 100,000$. Given the relatively low numbers of TORS, the robot is likely to be used only in centers with a high volume of gynecologic, urologic, cardiothoracic, and general procedures for which its use will be extended. ${ }^{11,12}$ The cost per case for the disposable TORS equipment required is approximately $\$ 500$, which is comparable to the cost of harmonic, laser, or other endoscopic technologies. ${ }^{2}$

The cost effectiveness of TORS is only relevant if it has short-term oncologic and functional equivalence compared to other modalities. Long-term results for TORS are not yet available. Reports thus far, however, show that oncologic outcomes are at least equivalent. ${ }^{5,13}$ Additionally, patients are less likely to require a gastrostomy tube and tracheotomy, thereby reducing costs. ${ }^{2,9,14}$ Furthermore, Richmon et al has shown that TORS followed by a rapid discharge protocol has not shown any increase in complications. ${ }^{14}$

\section{Conclusion}

TORS is a valuable treatment in the head and neck cancer surgeon's armamentarium for OPSCC. As the epidemic of HPV-associated OPSCC continues and the number of patients with this disease increase, it is in the best interest of society at large to figure out which treatments provide the best quality of life for patients, with the least cost. Based on the studies available, TORS for oropharyngeal cancer appears to be cost-effective compared to other available modalities. However, the current literature lacks large studies and long-term follow-up. Factors that make TORS more cost-effective are decreased hospital stay, increased chance of finding an unknown primary and thus potentially avoiding adjuvant treatment, and decreased rates of gastrostomy tube and tracheotomy. Elements making it less cost-effective are the capital investment and amortization of the robot as well as the cost of robot-related disposable equipment. The methods for calculating cost have varied widely. Future 
cost-analyses would benefit from a consistent method of cost calculation. Ideally, the calculated cost should include the actual reimbursement received by the hospital for all associated consultant and ancillary charges, including for readmissions within 90 days of treatment. In addition, the patient's decreased productivity during recovery should be taken into account, given the time to return to work may differ depending on treatment modality. Subsequent studies that include these factors will be able to more definitively show where TORS stands in terms of cost effectiveness in the treatment of OPSCC.

\section{Disclosure}

The authors report no conflicts of interest in this work.

\section{References}

1. Mcleod IK, Melder PC. Da Vinci robot-assisted excision of a vallecular cyst: a case report. Ear Nose Throat J. 2005;84(3):170-172.

2. Richmon JD, Quon H, Gourin CG. The effect of transoral robotic surgery on short-term outcomes and cost of care after oropharyngeal cancer surgery. Laryngoscope. 2014;124(1):165-171.

3. ECRI Institute. Health Technology Forecast: Transoral Robotic Surgery (TORS) for Treating Head and Neck Cancer; 2013. Available from: https://www.ecri.org/Documents/Sample_Reports/Health_ Technology_Forecast.pdf. Accessed July 7, 2014.

4. Chaturvedi AK, Engels EA, Pfeiffer RM, et al. Human papillomavirus and rising oropharyngeal cancer incidence in the United States. $J$ Clin Oncol. 2011;29(32):4294-4301.
5. Moore EJ, Olsen SM, Laborde RR, et al. Long-term functional and oncologic results of transoral robotic surgery for oropharyngeal squamous cell carcinoma. Mayo Clin Proc. 2012;87(3):219-225.

6. Dziegielewski PT, Teknos TN, Durmus K, et al. Transoral robotic surgery for oropharyngeal cancer: long-term quality of life and functional outcomes. JAMA Otolaryngol Head Neck Surg. 2013;139(11): 1099-1108.

7. The World Bank. Health Expenditure, Total (\% of GDP): The World Bank Group; 2014. Available from: data.worldbank.org/indicator/ SH.XPD.TOTL.ZS. Accessed July 7, 2014.

8. Moore EJ, Hinni ML, Olsen KD, Price DL, Laborde RR, Inman JC. Cost considerations in the treatment of oropharyngeal squamous cell carcinoma. Otolaryngol Head Neck Surg. 2012;146(6):946-951.

9. Hammoudi K, Pinlong E, Kim S, Bakhos D, Morinière S. Transoral robotic surgery versus conventional surgery in treatment for squamous cell carcinoma of the upper aerodigestive tract. Head Neck. Epub ahead of print May 2014.

10. Byrd JK, Smith KJ, de Almeida JR, et al. Transoral robotic surgery and the unknown primary: a cost-effectiveness analysis. Otolaryngol Head Neck Surg. 2014;150(6):976-982.

11. Bhayani MK, Holsinger FC, Lai SY. A shifting paradigm for patients with head and neck cancer: transoral robotic surgery (TORS). Oncology (Williston Park). 2010;24(11):1010-1015.

12. Weinstein GS, O’Malley BW Jr, Desai SC, Quon H. Transoral robotic surgery: does the ends justify the means? Curr Opin Otolaryngol Head Neck Surg. 2009;17(2):126-131.

13. Li RJ, Richmon JD. Transoral endoscopic surgery: new surgical techniques for oropharyngeal cancer. Otolaryngol Clin North Am. 2012; 45(4):823-844.

14. Richmon JD, Feng AL, Yang W, Starmer H, Quon H, Gourin CG. Feasibility of rapid discharge after transoral robotic surgery of the oropharynx. Laryngoscope. 2014.
Robotic Surgery: Research and Reviews

\section{Publish your work in this journal}

Robotic Surgery: Research and Reviews is an international, peer reviewed, open access, online journal publishing original research, commentaries, reports, and reviews on the theory, use and application of robotics in surgical interventions. Articles on the use of supervisory-controlled robotic systems, telesurgical devices, and shared-control systems are

\section{Dovepress}

invited. The manuscript management system is completely online and includes a very quick and fair peer review system, which is all easy to use. Visit http://www.dovepress.com/testimonials.php to read real quotes from published authors. 